\title{
Source Parameters of Regional Earthquakes in Taiwan: 1999-2000 Including the Chi-Chi Earthquake Sequence
}

\author{
Honn $\mathrm{Kao}^{1, \star}$, Yu-Hua Liu${ }^{2}$, Wen-Tzong Liang ${ }^{2}$, and Wang-Ping Chen ${ }^{3}$
}

(Manuscript received 12 April 2002, in final form 8 July 2002)

\begin{abstract}
We report source parameters of 277 earthquakes that occurred during 1999-2000 in the Taiwan region, a time period dominated by the Chi-Chi earthquake sequence. We used an improved CMT inversion algorithm to handle high background noise and structural heterogeneity from complex tectonic settings near Taiwan. We also searched the best fitting solutions at an increment of $1 \mathrm{~km}$ of focal depths for all events that occurred after the Chi-Chi main shock on September 20, 1999. In general, central and northern Taiwan was seismically quiet before the Chi-Chi main shock. Aftershocks of this sequence dominated the regional seismicity for approximately 3 months afterward. The Chi-Chi sequence consists of a wide range of focal mechanisms, but the overall pattern is a west-verging thrust block bound by strike-slip tear faults near the northern and southern termini of the surface rupture - the Chelungpu fault. Seismicity outside of the source zone of the Chi-Chi sequence generally agrees with known active tectonics from previous studies. Nonetheless, under high elevations of the eastern Central Ranges, there are several intriguing cases of changing focal mechanisms from normal faulting near the surface to thrust at depth. To facilitate further research of the Chi-Chi sequence, results of our inversion and original waveforms from the Broadband Array in Taiwan for Seismology (BATS) of 170 events within this sequence are ready for distribution on CD-ROM. The dataset is also online at the BATS web site (http://bats.earth.sinica. edu.tw/Chi-Chi_CMT).
\end{abstract}

(Key words: BATS, Earthquake source parameters, Waveform inversion, Chi-Chi earthquake sequence)

\footnotetext{
${ }^{1}$ Geological Survey of Canada, Pacific Geoscience Centre, Sidney, BC V8L 4B2, Canada

${ }^{2}$ Institute of Earth Sciences, Academia Sinica, Taipei, Taiwan, ROC

${ }^{3}$ Department of Geology and the NSF Mid-America Earthquake (MAE) Center, University of Illinois, Urbana, IL 61801, USA

* Corresponding author address: Dr. Honn Kao, Geological Survey of Canada, Pacific Geoscience Centre, Box 6000, 9860 West Saanich Road, Sidney, BC V8L 4B2, Canada

E-mail: hkao@nrcan.gc.ca
} 


\section{INTRODUCTION}

Development of the centroid-moment-tensor (CMT) inversion in the early 1980's allowed the systematic determination of source parameters for many large- and moderate-sized earthquakes on a global scale (e.g., Dziewonski et al. 1981; Kawakatsu et al. 1995; Sipkin, 1982). Later on, knowledge of seismic velocity structures on regional scale and establishment of regional broadband networks facilitated the routine application of CMT inversion to regional earthquakes (e.g., Dreger and Helmberger, 1993; Lay et al. 1994; Zhu and Helmberger, 1996; Pasysnos et al. 1996).

Taiwan has one of the highest seismicity in the world. Since late 1994, the Broadband Array in Taiwan for Seismology (BATS, Fig. 1) has enabled the systematic determination of reliable source parameters for regional earthquakes using the CMT technique (Kao et al. 1998, 2001; Kao and Jian, 1999, 2001).

This paper is a continuing effort to systematically report source parameters of regional events using waveforms recorded by the BATS. This report includes earthquakes that occurred in 1999 and 2000 with most of them associated with the devastating (Mw 7.5) Chi-Chi earthquake sequence of September 20, 1999.

In response to high interest in seismic data from the Chi-Chi sequence, the Data Management Center of the Institute of Earth Sciences (DMC-IES), Academia Sinica, has put in considerable efforts to prepare event-oriented files according to the Standard for Exchange of Earthquake Data (SEED) of the Federation of Digital Seismographic Network (FDSN). To this end, this paper also serves the purpose of announcing the availability of SEED volumes recorded by BATS through either CD-ROM or the Internet. We made this dataset complimentary to the public in the hope to facilitate further research of the Chi-Chi sequence.

\section{DATA AND ANALYSIS}

BATS has been in constant expansion, with 12 permanent stations established prior to the occurrence of the Chi-Chi earthquake. Currently, it has 13 permanent stations over an aperture of $\sim 450 \mathrm{~km}$ by $\sim 350 \mathrm{~km}$ (Fig. 1). Each station consists of a state-of-the-art 24-bit data logger (Quanterra Q-series) with two sensors, one very-broadband Streckeisen STS-1 or STS-2 seismometer and one Terra Technology SSA-320 accelerograph. Each station sends continuous data stream to the nearest Internet node via either dedicated phone line or microwave. The data packets are then transmitted back to DMC-IES in Taipei for further processing. The BATS website (http://bats.sinica.edu.tw) describes detailed configuration of the array and its current operations.

The DMC-IES is responsible for processing, quality control, storage/archiving, and data distribution from BATS. We also deposit a replica to the Data Management Center of the Incorporated Research Institutions for Seismology (DMC of IRIS) for their distribution. Currently, the DMC-IES is collaborating with the Data Center of the Ocean Hemisphere Project (OHP), Tokyo University, to establish a joint interface that offers direct data request and retrieval from both BATS and OHP data servers. Technical aspects of this interface, named NINJA, are fully described in a recent report (Takeuchi et al. 2002). 


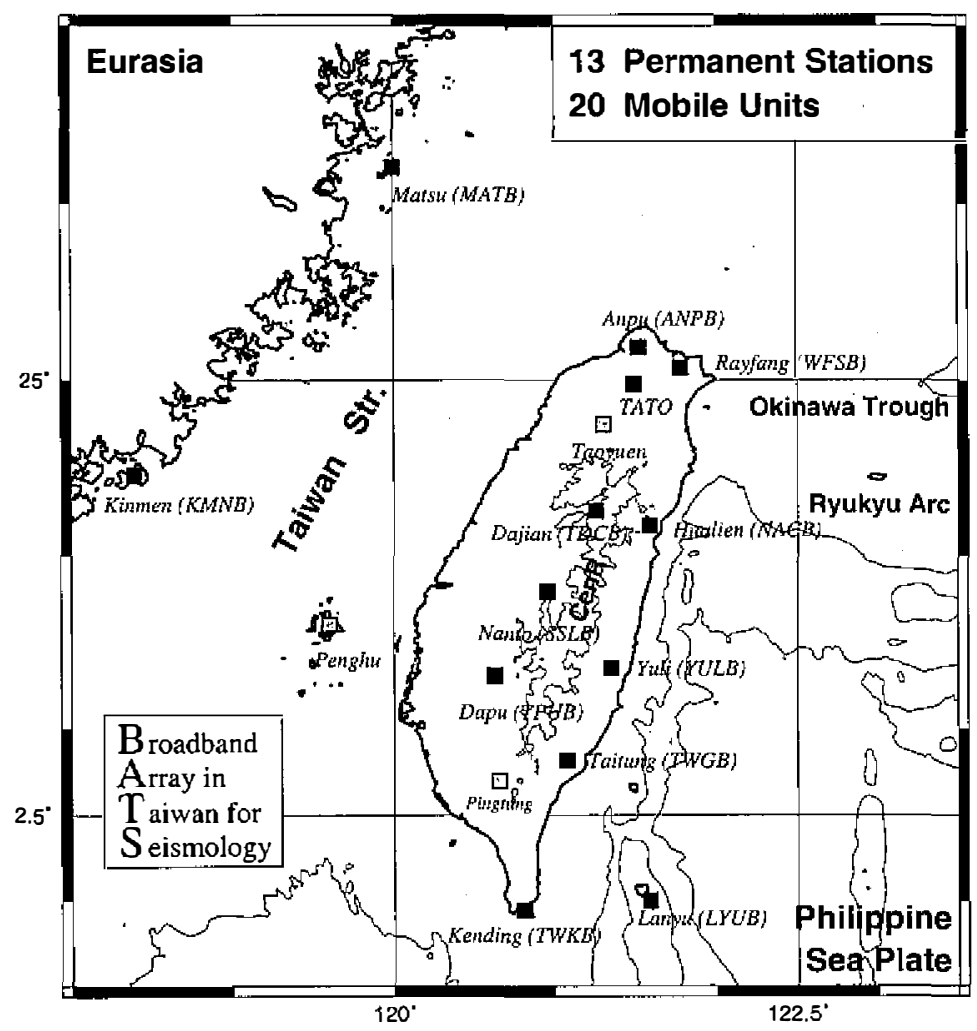

Fig. 1. Map showing the configuration of the Broadband Array in Taiwan for Seismology (BATS). Solid and gray squares show stations currently in operation and under construction, respectively. In addition to the permanent stations, BATS includes 20 mobile units that can be deployed for specific tasks. The topographic contours are in $2000 \mathrm{~m}$ interval. CenR: the Central Range.

Our CMT algorithm begins with a background-noise evaluation to determine the frequency band of the inversion. By comparing the power spectra of data in two windows spanning 300 s before and after the $P$ first arrival, the low-frequency cut-off is usually at $\sim 0.02 \mathrm{~Hz}$ (Kao et al. 1998). We set the high-frequency cut-off at $0.05-0.08 \mathrm{~Hz}$ to reduce the scattering effect and to avoid the calculation of high frequency portion of the synthetics. We also apply different velocity models for different station-source pairs to allow lateral heterogeneity (Kao et al. 1998; Kao and Jian, 1999).

Previously, the CMT inversion seeks the best-fitting focal depth at an interval of $3 \mathrm{~km}$. We further refined this interval to $1 \mathrm{~km}$ for all events that occurred after the main shock of the Chi-Chi sequence, so uncertainties in focal depths can be fully explored. For all source parameters, we explore the model space through forward modeling. The uncertainties reported in Table 1 correspond to values that cause a 5-10\% increase of the overall root-mean-square (RMS) misfit between observed and synthetic waveforms. 
Table 1. Source Parametrs of Earthquakes.

\begin{tabular}{|c|c|c|c|c|c|c|c|c|c|c|c|}
\hline No & Origin Time ${ }^{2}$ & Lat. $^{2}$ & Long. ${ }^{2}$ & Depth & $\mathrm{M}_{\mathrm{w}}{ }^{3}$ & Strike $^{3}$ & $\operatorname{Dip}^{3}$ & Rake $^{3}$ & $E^{4}$ & $\varepsilon^{4}$ & Class \\
\hline 1 & $99 / 01 /[2 / 06: 24: 16.2$ & 23.30 & 121.75 & $21 \pm 5$ & $4.12 \pm 0.21$ & $276 \pm 4$ & $19 \pm 9$ & $166 \pm 6$ & 0.438 & 21.10 & $\overline{B 2}$ \\
\hline 2 & $99 / 02 / 13 / 21: 47: 57.4$ & 23.89 & 122.47 & $24 \pm 4$ & $4.41 \pm 0.22$ & $292 \pm 9$ & $27 \pm 5$ & $128 \pm 7$ & 0.536 & 13.53 & $\mathrm{C} 2$ \\
\hline 3 & $99 / 02 / 22 / 13: 48: 58.0$ & 23.98 & 122.65 & $30 \pm 6$ & $5.71 \pm 0.29$ & $306 \pm 13$ & $32 \pm 10$ & $141 \pm 7$ & 0.482 & 6.61 & B1 \\
\hline 4 & 99/02/28/04:04:44.7 & 24.36 & 121.97 & $24 \pm 5$ & $4.15 \pm 0.21$ & $108 \pm 3$ & $40 \pm 3$ & $-15 \pm 10$ & 0.386 & 10.81 & B2 \\
\hline 5 & $99 / 03 / 05 / 21: 12: 27.4$ & 23.25 & 120.48 & $18 \pm 3$ & $4.02 \pm 0.20$ & $175 \pm 12$ & $42 \pm 3$ & $83 \pm 3$ & 0.395 & 29.58 & B3 \\
\hline 6 & $99 / 03 / 26 / 14: 34: 09.5$ & 24.74 & 122.91 & $126 \pm 9$ & $4.36 \pm 0.22$ & $42 \pm 3$ & $14 \pm 8$ & $-156 \pm 9$ & 0.496 & 27.01 & B3 \\
\hline 7 & 99/04/04/04:59:02.6 & 24.88 & 121.94 & $102 \pm 8$ & $5.01 \pm 0.25$ & $106 \pm 7$ & $11 \pm 4$ & $-126 \pm 10$ & 0.478 & 17.94 & B2 \\
\hline 8 & 99/04/04/13:53:01.1 & 24.87 & 122.21 & $12 \pm 3$ & $4.47 \pm 0.22$ & $97 \pm 13$ & $5 \pm 14$ & $-63 \pm 16$ & 0.639 & 20.99 & $\mathrm{C} 2$ \\
\hline 9 & 99/04/06/16:14:39.9 & 24.76 & 122.24 & $21 \pm 5$ & $4.31 \pm 0.22$ & $44 \pm 6$ & $77 \pm 7$ & $179 \pm 6$ & 0.646 & 2.29 & $\mathrm{Cl}$ \\
\hline 10 & $99 / 04 / 10 / 07: 52: 23.5$ & 24.09 & 121.71 & $18 \pm 3$ & $4.47 \pm 0.22$ & $239 \pm 3$ & $43 \pm 7$ & $126 \pm 3$ & 0.481 & 22.93 & B2 \\
\hline 11 & $99 / 0$ & 22.05 & 120.02 & $60 \pm 6$ & $4 \pm 0.23$ & $132 \pm 6$ & $51 \pm 10$ & $-165 \pm 3$ & 0.645 & 10.74 & $\mathrm{C} 2$ \\
\hline 12 & 99/05/ & 21.24 & 122.03 & $24 \pm 3$ & $4.70 \pm 0.23$ & $4 \pm 25$ & $12 \pm 5$ & $-65 \pm 25$ & 0.549 & 12.47 & $\mathrm{C} 2$ \\
\hline 13 & 03:24.4 & 24.74 & 121.89 & $12 \pm 3$ & $5.01 \pm 0.25$ & $73 \pm 9$ & $20 \pm 3$ & $-52 \pm 8$ & 0.513 & 6.95 & $\mathrm{Cl}$ \\
\hline 14 & $99 / 05$ & 23.95 & 121.09 & $24 \pm 4$ & $3.65 \pm 0.18$ & $207 \pm 8$ & $44 \pm 3$ & $93 \pm 6$ & 0.376 & 34.15 & B3 \\
\hline 15 & 13/15:05:50.8 & 24.28 & 122.61 & $48 \pm 5$ & $4.23 \pm 0.21$ & $26 \pm 7$ & $38 \pm 3$ & $110 \pm 11$ & 0.393 & 15.59 & B2 \\
\hline 16 & $99 / 0$ & 22.17 & 121.40 & $72 \pm 6$ & \pm 0.23 & $296 \pm 15$ & $32 \pm 8$ & $44 \pm 10$ & 0.469 & 12.61 & B2 \\
\hline 17 & $99 / 06 /$ & 24.16 & 121.69 & $33 \pm 3$ & $3.94 \pm 0.20$ & $248 \pm 6$ & $33 \pm 5$ & $142+7$ & 0.422 & 17.79 & B2 \\
\hline 18 & $99 / 06 / 03$ & 24.40 & 122.49 & $63 \pm 6$ & $5.24 \pm 0.26$ & $66 \pm 3$ & $37 \pm 8$ & $179 \pm 9$ & 0.389 & 32.73 & B3 \\
\hline 19 & $99 / 06 / 1$ & 23.73 & 121.73 & $30 \pm 5$ & $4.56 \pm 0.23$ & $292 \pm 8$ & $46 \pm 3$ & $20 \pm 6$ & 0.418 & 48.29 & B4 \\
\hline 20 & $99 / 06 / 2$ & 22.84 & 121.36 & $21 \pm 4$ & $4.12 \pm 0.21$ & $342 \pm 5$ & $43 \pm 3$ & $74 \pm 6$ & 0.372 & 13.68 & B2 \\
\hline 21 & $99 / 06$ & 21.50 & 119.89 & $48 \pm 7$ & $4.62 \pm 0.23$ & $241 \pm 3$ & $44 \pm 3$ & $125 \pm 3$ & 0.557 & 11.28 & $\mathrm{C} 2$ \\
\hline 22 & 99/07/ & 21.19 & 120.22 & $27 \pm 4$ & $3.94 \pm 0.20$ & $54 \pm 3$ & $49 \pm 8$ & $-23 \pm 3$ & 0.663 & 5.19 & $\mathrm{C} 1$ \\
\hline 23 & 99/07/ & 23.32 & 120.74 & $24 \pm 3$ & $4.62 \pm 0.23$ & $188 \pm 7$ & $33 \pm 5$ & $54 \pm 8$ & 0.458 & 21.04 & B2 \\
\hline 24 & $6: 29.2$ & 23.31 & 120.74 & $18 \pm 4$ & $4.21 \pm 0.21$ & $216 \pm 7$ & $43 \pm 3$ & $95 \pm 3$ & 0.494 & 11.67 & B2 \\
\hline 25 & $99 / 07 / 08 / 08$ & 23.97 & 122.42 & $18 \pm 4$ & $4.09 \pm 0.20$ & $287 \pm 3$ & $24 \pm 3$ & $136 \pm 9$ & 0.543 & 17.77 & $\mathrm{C} 2$ \\
\hline 26 & $99 / 03$ & 23.32 & 120.75 & $18 \pm 3$ & $=0.21$ & $185 \pm 3$ & $45 \pm 6$ & $15+3$ & 0.489 & 6.63 & B1 \\
\hline 27 & $99 / 07$ & 22.97 & 122.44 & $18 \pm 3$ & $4.19 \pm 0.21$ & $341 \pm 8$ & $59 \pm 8$ & $14 \pm 8$ & 0.671 & 35.84 & C3 \\
\hline 28 & $99 / 07$ & 24.60 & 121.84 & $75 \pm 6$ & $4.38 \pm 0.22$ & $347 \pm 4$ & $33 \pm 3$ & $3 \pm 10$ & 0.49 & 20.83 & B2 \\
\hline 29 & $99 / 07 / 23 / 14: 30: 28.0$ & 23.40 & 120.52 & $12 \pm 3$ & $3.60 \pm 0.18$ & $284 \pm 9$ & $75 \pm 9$ & $165 \pm 5$ & 0.532 & 4.35 & Cl \\
\hline 30 & $99 / 08 / 04 / 21: 03: 51.5$ & 22.79 & 121.14 & $21 \pm 4$ & $4.43 \pm 0.22$ & $68 \pm 25$ & $24 \pm 13$ & $72 \pm 25$ & 0.498 & 21.68 & B2 \\
\hline 31 & $99 / 08 / 05$ & 24.93 & 119.18 & $12 \pm 3$ & \pm 0.20 & $350 \pm 6$ & $62 \pm 3$ & $-170 \pm 3$ & 0.556 & 12.74 & C2 \\
\hline 32 & 42.8 & 23.11 & 121.02 & $2 \mathrm{I} \pm 3$ & 0.21 & $271 \pm 7$ & $56 \pm 9$ & $64 \pm 9$ & .447 & 16.61 & B2 \\
\hline 33 & $18: 20.8$ & 22.44 & 121.82 & $21 \pm 4$ & \pm 0.26 & $199 \pm 3$ & $26 \pm 3$ & $127 \pm 9$ & 0.417 & 37.07 & B3 \\
\hline 34 & $44: 07.2$ & 21.13 & 120.15 & $54 \pm 8$ & $4.55 \pm 0.23$ & $61 \pm 3$ & $34 \pm 18$ & $4 \pm 5$ & 0.534 & 37.12 & C3 \\
\hline 35 & 99/09/16/16:12:01.1 & 21.31 & 120.12 & $42 \pm 5$ & $4.70 \pm 0.23$ & $250 \pm 3$ & $52 \pm 4$ & $143 \pm 9$ & 0.483 & 4.68 & B1 \\
\hline 36 & $99 / 09$ & 23.42 & 120.62 & $18 \pm 4$ & $3.81 \pm 0.19$ & $327 \pm 9$ & $35 \pm 5$ & $59 \pm 7$ & 0.542 & 34.28 & C3 \\
\hline 37 & 10.8 & 23.79 & 120.88 & $17 \pm 6$ & $6.22 \pm 0.31$ & $25 \pm 5$ & $32 \pm 4$ & $97 \pm 12$ & 0.572 & 23.27 & C2 \\
\hline 38 & $99 / 09 / 20 / 18: 11: 52.7$ & 23.85 & 121.06 & $24 \pm 4$ & $6.10 \pm 0.31$ & $303 \pm 3$ & $49 \pm 10$ & $7 \pm 9$ & 0.529 & 1.19 & $\mathrm{Cl}$ \\
\hline 39 & $99 / 09 / 20 / 18: 16: 16.5$ & 23.84 & 121.04 & $20 \pm 6$ & $6.11 \pm 0.31$ & $337 \pm 9$ & $38 \pm 3$ & $65 \pm 13$ & 0.441 & 20.36 & B2 \\
\hline 40 & 99/09/20/19:40:31.3 & 23.55 & 120.91 & $15 \pm 8$ & $5.41 \pm 0.27$ & $339 \pm 6$ & $63 \pm 3$ & $15 \pm 4$ & 0.544 & 26.74 & C3 \\
\hline 41 & $: 48.3$ & 23.77 & 121.33 & $12 \pm 5$ & $4.84 \pm 0.24$ & $348 \pm 5$ & $24 \pm 3$ & $119 \pm 9$ & .580 & 29.78 & C3 \\
\hline 42 & $99 / 09$ & 23.60 & 120.82 & $19 \pm 5$ & \pm 0.31 & $242 \pm 6$ & $55 \pm 3$ & $58 \pm 7$ & 0.497 & 21.67 & B2 \\
\hline 43 & 99/09/20/23:18:13.0 & 23.46 & 120.89 & $12 \pm 5$ & $4.78 \pm 0.24$ & $353 \pm 3$ & $53 \pm 3$ & $-5 \pm 8$ & 0.497 & 31.56 & B3 \\
\hline 44 & $99 / 09 / 21 / 00: 45: 40.0$ & 23.88 & 120.99 & $21 \pm 6$ & $4.51 \pm 0.23$ & $10 \pm 9$ & $37 \pm 5$ & $64 \pm 5$ & 0.466 & 7.24 & Bl \\
\hline 45 & $99 / 09 / 21 / 01$ & 23.72 & 120.75 & $13 \pm 5$ & $4.65 \pm 0.23$ & $103 \pm 10$ & $20 \pm 3$ & $-173 \pm 9$ & 0.564 & 0.04 & $\mathrm{Cl}$ \\
\hline 46 & $99 / 09 / 21 / 02$ & 23.92 & 121.01 & $18 \pm 6$ & $4.08 \pm 0.20$ & $334 \pm 7$ & $41 \pm 3$ & $62 \div 8$ & 0.398 & 16.96 & B2 \\
\hline 47 & 99/09/21/02:34:35.6 & 23.65 & 120.83 & $15 \pm 5$ & $4.48 \pm 0.22$ & $336 \pm 3$ & $55 \pm 7$ & $3 \pm 6$ & 0.535 & 16.28 & C2 \\
\hline 48 & $99 / 09 / 21 / 02: 45: 40.2$ & 24.01 & $12 \mathrm{I} .04$ & $18 \pm 6$ & $4.41 \pm 0.22$ & $98 \pm 5$ & $53 \pm 3$ & $-166 \pm 7$ & 0.494 & 36.44 & B3 \\
\hline 49 & 99/09/21/03:01:29.5 & 24.16 & 121.59 & $17 \pm 5$ & $4.62 \pm 0.23$ & $205 \pm 8$ & $28 \pm 3$ & $37 \pm 20$ & 0.485 & 26.05 & B3 \\
\hline 50 & 99/09/21/03:25:59.5 & 23.72 & 120.77 & $12 \pm 5$ & $4.52 \pm 0.23$ & $84 \pm 25$ & $13 \pm 3$ & $174 \pm 25$ & 0.585 & 10.55 & $\mathrm{C} 2$ \\
\hline 51 & $99 / 09 / 21 / 03: 31: 48.1$ & 24.00 & 121.01 & $24 \pm 6$ & $4.50 \pm 0.23$ & $355 \pm 5$ & $46 \pm 3$ & $-16 \pm 9$ & 0.385 & 10.36 & B2 \\
\hline 52 & $99 / 09 / 21 / 06: 36: 27.2$ & 24.03 & 121.02 & $23 \pm 5$ & $3.98 \pm 0.20$ & $338 \pm 3$ & $43 \pm 5$ & $-16 \pm 3$ & 0.398 & 10.21 & B2 \\
\hline 53 & 99/09/21/07:06:01.7 & 23.80 & 121.40 & $13 \pm 5$ & $4.67 \pm 0.23$ & $123 \pm 3$ & $52 \pm 6$ & $-136 \pm 3$ & $0.5 ! 5$ & 5.20 & Cl \\
\hline 54 & $99 / 09 / 21 / 07: 40: 52.5$ & 24.23 & 121.03 & $27 \pm 6$ & $4.34 \pm 0.22$ & $345 \pm 8$ & $42 \pm 6$ & $-34 \pm 8$ & 0.492 & 31.84 & B3 \\
\hline 55 & $99 / 09 / 21 / 07: 46: 04.6$ & 24.10 & 121.13 & $26 \pm 6$ & $4.68 \pm 0.23$ & $24 \pm 4$ & $62 \pm 3$ & $-6 \pm 3$ & 0.433 & 25.59 & B3 \\
\hline 66 & & & & & & & & & 0.4 & & \\
\hline
\end{tabular}




\section{(Table 1 . continued)}

\begin{tabular}{|c|c|c|c|c|c|c|c|c|c|c|c|}
\hline & & & & & & & & & & & \\
\hline & & & & $7 \pm 4$ & & & & & & & \\
\hline & $/ 09 / 21 / 09: 36: 10.3$ & & & $1 \pm 5$ & & & & & & & \\
\hline & $/ 09 / 21 / 11: 07: 41.4$ & 3.67 & 120.84 & $1 \pm 4$ & $43 \pm 0.22$ & $57 \pm 3$ & & $7 \pm 9$ & & & \\
\hline & & & & $1 \pm 6$ & & & & & & & \\
\hline & & & & $3 \pm 6$ & & & & & & & \\
\hline & & & & $19 \pm 4$ & & & $i \neq 7$ & & & & \\
\hline & $4: 32: 13.3$ & 24.09 & 21.36 & $22 \pm 5$ & & $271 \pm 3$ & $0 \pm 3$ & & 522 & 2.48 & \\
\hline & 3.0 & 24.32 & 120.85 & $12 \pm 5$ & \pm 0.22 & $290 \pm 11$ & $1 \pm 4$ & $0 \pm 13$ & 539 & 4.54 & \\
\hline & & 23.62 & & $12 \pm 5$ & & & & & 527 & & \\
\hline & & & & $21 \pm 6$ & & & & & & & \\
\hline & 7.6 & 24.18 & 20.93 & $13 \pm 5$ & & $44 \pm 3$ & $63 \pm 7$ & $-26 \pm 10$ & 483 & 0.16 & \\
\hline & 5.0 & 23.88 & 1.28 & $34 \pm 7$ & & $6 \pm 5$ & & & & 5.85 & \\
\hline & 5.3 & 24.18 & & $16 \pm 7$ & & $1 \pm 3$ & $6 \pm 4$ & & & & \\
\hline & & & & & & & & & & & \\
\hline & 2.9 & 23.76 & 02 & $24 \pm 8$ & & $20 \pm 12$ & & & & 90 & \\
\hline & 9.6 & & 39 & $11 \pm 5$ & & & & & & & \\
\hline & .3 & & & $12 \pm 5$ & & & & & & & \\
\hline & .6 & & 83 & $12 \pm 5$ & & $44 \pm 3$ & $3 \pm 9$ & & 499 & & \\
\hline & & & & $31 \pm 7$ & & & & & & & \\
\hline & 5 & & & $12 \pm 4$ & & \pm 12 & & & & & \\
\hline & 1.1 & & & $23 \pm 6$ & & & & & & & \\
\hline & & & & $18 \pm 5$ & & & & & & & \\
\hline & .5 & & & 4 & & \pm 5 & & & & .81 & \\
\hline & .0 & 97 & & 18 & & $30 \pm 3$ & & & & 8.08 & \\
\hline & 30 & & & & & & & & & 3.99 & \\
\hline & & & & & & & & & & & \\
\hline & 7 & & & $18=$ & & & & & & & \\
\hline & $99 /$ & & & $=5$ & & & & & & & \\
\hline & & & & $13 \pm 6$ & & & & & & & \\
\hline & & & & $7 \pm 6$ & & & 28 & & & 2.02 & \\
\hline & & & & 16 & & & & & & & \\
\hline & & & & 5 & & & & & & & \\
\hline & & & & & & & & & & & \\
\hline & & & & & & & & & & & \\
\hline & & & & & & & & & & & \\
\hline & & & & $t 5$ & & & & & & & \\
\hline & & & & 5 & & & & & & & \\
\hline & & & & & & & & & & & \\
\hline & & & & & & & & & & & \\
\hline & & & & & & & & & & & \\
\hline & & & & $25 \pm 7$ & & & & & & & \\
\hline & & & & $0 \pm 6$ & & & & & & & \\
\hline & & & & \pm 5 & & & & & & & \\
\hline & & & & & & & & & & & \\
\hline & & & & $18 \pm 8$ & & & & & & 17.50 & \\
\hline & & & & & & & & & & & \\
\hline & & & & & & & & & & & \\
\hline & & & & & & & & & & & \\
\hline & & & & $15 \pm 5$ & & & $36 \pm 5$ & & & 10.36 & \\
\hline & & & & $28 \pm 6$ & & & & & & 9.01 & \\
\hline & & & & & & & & & & & \\
\hline & & & & & & & & & & 24.39 & \\
\hline & & & & & & & & & & & \\
\hline & & 23.18 & & $15 \pm 4$ & & & $15 \pm 7$ & & 492 & 9.08 & \\
\hline & 28.6 & & & $27 \pm 6$ & & $138 \pm 4$ & & $101 \pm 6$ & & 0.02 & \\
\hline & & & & 20 & & & & & & 5.51 & \\
\hline & 99/09/28/23:0 0 :25.8 & 23.67 & & $21 \pm 5$ & $4.23 \pm 0.21$ & $65 \pm 3$ & $63 \pm 3$ & & 0.543 & 11.10 & \\
\hline
\end{tabular}


(Table 1 . continued)

\begin{tabular}{|c|c|c|c|c|c|c|c|c|c|c|c|}
\hline & & & & & & & & & & & \\
\hline & $9 / 09 / 29 / 00: 22: 12.5$ & .61 & 20.86 & $1 \pm 8$ & $97 \pm 0.20$ & $38 \pm 6$ & & & & & \\
\hline & & .86 & & & & & & & & & \\
\hline & & & & & & & & & & 67 & \\
\hline & & .44 & & $2 \pm 4$ & & $3 \pm 3$ & & & & 25.43 & \\
\hline & $/ 09 / 30 / 09: 28: 12.7$ & .56 & 20.88 & $7 \pm 5$ & & $339 \pm 8$ & & & & .40 & \\
\hline & & .53 & 20.95 & $3 \pm 8$ & & $35 \pm 3$ & & & & 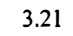 & \\
\hline & & 68 & & $4 \pm 8$ & & $5 \pm 3$ & & & & .80 & \\
\hline & /09/30/09:52:3 & 3.29 & 20.64 & $2 \pm 5$ & & $23 \pm 10$ & & & & 5.13 & \\
\hline & $/ 09 / 30 / 10: 20: 4$ & .64 & 20.86 & \pm 11 & & $69 \pm 4$ & & & & 2.12 & \\
\hline & 2.3 & 4.06 & 21.53 & $8 \pm 6$ & & $87 \pm 10$ & $24 \pm 10$ & & 24 & .39 & \\
\hline & & 69 & 20.91 & $1 \pm 6$ & & & & & & .73 & \\
\hline & 2.1 & .69 & 20.93 & $3 \pm 4$ & & $63 \pm 8$ & & & & 0.89 & \\
\hline & & 3.97 & 121.34 & $2 \pm 11$ & & $102 \pm 12$ & & & & .04 & \\
\hline & & .89 & & $2 \pm 6$ & & 355 & & & & .87 & \\
\hline & & .96 & & $0 \pm 5$ & & & & & & 1.20 & \\
\hline & & 2.18 & 120.92 & $24 \pm 6$ & & & & & 55 & 4.55 & \\
\hline & & 2.11 & 121.30 & $28 \pm 5$ & & $219 \pm 3$ & & & & .45 & \\
\hline & 2.9 & 3.57 & & $14 \pm 6$ & & & & & & 3.96 & \\
\hline & & .18 & & $7 \pm 6$ & & $348 \pm 3$ & & & & & \\
\hline & & .79 & & $8 \pm 5$ & & 15 & & & & 34 & \\
\hline & & 3.84 & 00 & $15 \pm 5$ & & & & & & & \\
\hline & & 3.96 & 03 & $20 \pm 5$ & & & & & & 37 & \\
\hline & & 3.58 & & $3 \pm 5$ & & & & & & 41 & \\
\hline & & .99 & & $8 \pm 6$ & & & & & & .32 & \\
\hline & & 3.70 & 6 & $12 \pm 7$ & & $49+$ & & & & 11.81 & \\
\hline & & 3.99 & & & & & & & & 7.69 & \\
\hline & & 3.96 & & $15 \pm 6$ & & & & & & 59 & \\
\hline & & 3.99 & & $18 \pm 8$ & & ES & & & & 1.20 & \\
\hline & & 4.00 & & $15 \pm 4$ & & & & & & 84 & \\
\hline & & 4.04 & & \pm 7 & & & & & & & \\
\hline & & .95 & & 24 & & & & & & & \\
\hline & & .70 & & $4 \pm 5$ & & & & & & & \\
\hline & & 3.63 & & & & & & & & & \\
\hline & & 34 & & $2 \pm 6$ & & & & & & 69 & \\
\hline & & & & & & & & & & & \\
\hline & & & & & & & & & & .73 & \\
\hline & & .53 & & $21 \pm 5$ & & 2 & & & & & \\
\hline & & .53 & & $8 \pm 5$ & & & & & & 86 & \\
\hline & & & & & & & & & & & \\
\hline & & 0 & & & & & & & & .40 & \\
\hline & & & & & & & & & & & \\
\hline & & & & & & & & & & & \\
\hline & & & & & & & & & & & \\
\hline & & & & & & & & & & .91 & \\
\hline & & & & & & & & & & 85 & \\
\hline & & & & & & & & & & 3.39 & \\
\hline & & & & & & & & & & & \\
\hline & & & & & & & & & & .98 & \\
\hline & & & & & & & & & & & \\
\hline & & & & & & & & & & & \\
\hline & & & & & & & & & & 5.88 & \\
\hline & & 3.29 & & & & & & & & 96 & \\
\hline & & & & & & & & & & 90 & \\
\hline & & & & & & & & & & & \\
\hline & & & & & & & & & & & \\
\hline 71 & & & & & & & & & & .22 & \\
\hline 2 & 99/11/13/19:53:53.6 & 23.89 & 122.34 & $29 \pm 6$ & $4.33 \pm 0.23$ & $316 \pm 4$ & $26 \pm 8$ & $16: \pm 10$ & 0.595 & 9.08 & \\
\hline
\end{tabular}


(Table 1. continued)

\begin{tabular}{|c|c|c|c|c|c|c|c|c|c|c|c|}
\hline & & & & $15 \pm 4$ & & & \pm 3 & & & & \\
\hline & 99/11/17/07:35:09.9 & 4.02 & 20.64 & $14 \pm 5$ & $93 \pm 0.25$ & $228 \pm 3$ & $3 \pm 9$ & \pm \pm 10 & 487 & 0.24 & \\
\hline & $/ 11 / 20 / 03: 29: 35.6$ & .02 & 0.65 & $17 \pm 4$ & $32 \pm 0.22$ & $26 \pm 5$ & \pm 7 & & 456 & 2.86 & \\
\hline & $/ 11 / 26 / 10: 12: 30.9$ & .95 & 2.82 & $6 \pm 6$ & $2 \pm 0.25$ & $7 \pm 3$ & \pm 3 & $79 \pm 7$ & & & \\
\hline & $/ 11 / 28 / 20: 42: 21.0$ & .31 & 0.92 & $15 \pm 8$ & $49 \pm 0.22$ & $0.43 \pm 3$ & $64 \pm 6$ & $11 \pm 19$ & & 1.90 & \\
\hline & $/ 11 / 28 / 21: 25: 50.2$ & .33 & 0.93 & $11 \pm 5$ & $4.60 \pm 0.23$ & $352 \pm 3$ & $5 \pm 6$ & $3 \pm 8$ & & & \\
\hline & /08/16:01:25.0 & .56 & 1.69 & \pm 5 & $4.00 \pm 0.20$ & $61 \pm 3$ & $2 \pm 3$ & $75 \pm 10$ & 427 & .97 & \\
\hline & 2.9 & 65 & 1.29 & $9 \pm 6$ & .23 & $156 \pm 3$ & $5 \pm 4$ & & & 10 & \\
\hline & & 3.77 & & \pm 8 & & $196 \pm 21$ & $28 \pm 8$ & & & & \\
\hline & & & & & & $242 \pm 3$ & $62 \pm 10$ & & & 7.90 & \\
\hline & & & & & & & & & & 82 & \\
\hline & & & & & & & & & & & \\
\hline & & & & $2 \pm 5$ & & & & & & & \\
\hline & & & & $1 \pm 5$ & & & $36 \pm 3$ & & & & \\
\hline & & & & & & & & & & & \\
\hline & & 3.99 & & \pm 4 & & & $5 \pm 3$ & & & & \\
\hline & & 4.36 & 0.93 & \pm 5 & & & $3 \pm 4$ & & & & \\
\hline & & 4.33 & & \pm 5 & & & $4 \pm 3$ & & & & \\
\hline & & 22.79 & & & & & & & & & \\
\hline & & & & & & & & & & & \\
\hline & & & & & & & & & & & \\
\hline & & 24.01 & & $26 \pm 7$ & 0.22 & $266 \pm 3$ & $16 \pm 7$ & $92 \pm 4$ & & & \\
\hline & & 3.22 & & $5 \pm 8$ & & $7 \pm 9$ & $\int \pm 0$ & $3 \pm 7$ & & 81 & \\
\hline & & & & $4 \pm 6$ & & & & $1 \pm 1$ & & 80 & \\
\hline & & & & & & & & & & & \\
\hline & & & & & & & & & & & \\
\hline & & & & & & & & & & & \\
\hline & & & & & & & & & & & \\
\hline & & & & $45 \pm 4$ & & $68 \pm 3$ & $3 \pm 4$ & & & & \\
\hline & & 3.31 & & $8 \pm 6$ & & $237 \pm 5$ & $3 \pm 4$ & $17 \pm 7$ & & 0.59 & \\
\hline & & 3.88 & & $1 \pm 6$ & & $176 \pm 7$ & $4 \pm 7$ & $4 \pm 12$ & & & \\
\hline & & & & $3 \pm 5$ & & & & & & & \\
\hline & & & & & & & & & & & \\
\hline & & & & & & & & & & & \\
\hline & & & & & & & & & & & \\
\hline & & & & & & & & & & & \\
\hline 9 & & 24.25 & & $18 \pm 8$ & & & & & & & \\
\hline & & 4.02 & & $28 \pm 7$ & & & & & & & \\
\hline & & 2.52 & & $36 \pm 7$ & & & & & & 28 & \\
\hline & & & & $=5$ & & 15 & & & & & \\
\hline & & & & & & & & & & & \\
\hline & & & & & & & & & & & \\
\hline & & & & & & & & & & & \\
\hline & & & & $18=$ & & & & & & & \\
\hline & & & & & & $201 \pm 3$ & $54 \pm 4$ & & & 5.99 & \\
\hline & & & & \pm 8 & & & & & & 58 & \\
\hline & & & & & & & & & & & \\
\hline & & & & & & & & & & & \\
\hline & & & & & & & & & & & \\
\hline & & & & & & & & & & & \\
\hline & & & & & & & & & & & \\
\hline & & & & & & & & & & & \\
\hline & & & & & & & & & & & \\
\hline & & & & & & & & & & & \\
\hline & & & & & & & & & & & \\
\hline & & & & & & & & & & & \\
\hline & & & & & & & & & & & \\
\hline & & & & & & & & & & & \\
\hline & & & & & & & & & & & \\
\hline & & & & & & & & & & 12.64 & \\
\hline & & & & & & & & & & & \\
\hline & & & & & & & & & & & \\
\hline & & & & & & & & & & & \\
\hline & & & & & & & & & & & \\
\hline
\end{tabular}


(Table 1 . continued)

\begin{tabular}{|c|c|c|c|c|c|c|c|c|c|c|c|}
\hline 237 & 00/07/17/06:50:39.4 & 24.07 & 121.79 & $15 \pm 6$ & $4.58 \pm 0.23$ & $218 \pm 9$ & $26 \pm 3$ & $68 \pm 18$ & 0.543 & 5.19 & $\mathrm{C} 1$ \\
\hline 238 & 00/07/19/04:46:33.4 & 24.12 & 121.76 & $15 \pm 5$ & $4.68 \pm 0.23$ & $214 \pm 7$ & $24 \pm 3$ & $54 \pm 17$ & & 10.60 & B2 \\
\hline 239 & $00 / 07 / 24 / 22: 10: 36.4$ & 24.71 & 122.48 & $110 \pm 7$ & $4.90 \pm 0.25$ & $189 \pm 9$ & $51 \pm 3$ & $7 \pm 5$ & 0.454 & 2.01 & 1 \\
\hline 240 & 00/07/28/20:28:07.7 & 23.41 & 120.93 & $12 \pm 5$ & $5.65 \pm 0.28$ & $354 \pm 3$ & $63 \pm 4$ & $11 \pm 9$ & 0.494 & 14.33 & B2 \\
\hline 41 & 00/08/02/08:34:32.0 & 23.85 & 121.02 & $18 \pm 5$ & $4.42 \pm 0.22$ & $347 \pm 3$ & $38 \pm 5$ & $67 \pm 10$ & 0.465 & 2.85 & B1 \\
\hline 242 & 00/08/05/02:32:27.5 & 24.18 & 122.51 & $39 \pm 5$ & $4.28 \pm 0.2 \mathrm{l}$ & $159 \pm 6$ & $46 \pm 5$ & $29 \pm 5$ & 0.436 & 8.23 & B1 \\
\hline 243 & 00/08/06/09:51:40:8 & 24.27 & 121. & $20 \pm 8$ & $4.71 \pm 0.24$ & $326 \pm 6$ & $18 \pm 5$ & $-155 \pm 8$ & 0.638 & 3.87 & $\mathrm{C} 1$ \\
\hline 244 & 00/08/07/03:1 & 23.34 & 120. & $11 \pm 5$ & 0.21 & $263 \pm 3$ & $27 \pm 3$ & $-177 \pm 3$ & 0.597 & 25.51 & 3 \\
\hline 245 & $00 / 08 / 12 / 12$ & 23.35 & 120.94 & $12 \pm 5$ & 0.21 & $259 \pm 15$ & $41 \pm 4$ & $168 \pm 21$ & 0.577 & 3.34 & $\mathrm{Cl}$ \\
\hline 246 & 00/08/20/06: & 23.06 & 120. & $13 \pm 6$ & \pm 0.22 & $263 \pm 4$ & $43 \pm 6$ & $-164=16$ & 0.576 & 30.29 & $\mathrm{C} 3$ \\
\hline 247 & $00 / 08 / 20 / 10$ & 23.08 & 120 & $12 \pm 5$ & 0.24 & $268 \pm 14$ & $38 \pm 7$ & $-160 \pm 23$ & 0.593 & 6.27 & $\mathrm{Cl}$ \\
\hline 248 & $00 / 08 / 20 / 1$ & 23.10 & 120 & $13 \pm 6$ & 0.23 & $162 \pm 13$ & $60 \pm 8$ & $-19 \pm 19$ & 0.538 & 3.53 & $\mathrm{C} 1$ \\
\hline 249 & $00 / 08 / 21 / 1$ & 22.95 & 120 & $12 \pm 5$ & 0.21 & $285 \pm 8$ & $40 \pm 6$ & $-145 \pm 5$ & 0.496 & 3.24 & B1 \\
\hline 250 & $00 / 08$ & 23.64 & 121.63 & $36 \pm 6$ & 0.26 & $14 \pm 4$ & $52 \pm 3$ & $46 \pm 6$ & 0.360 & 8.79 & $\mathrm{Bl}$ \\
\hline 51 & $00 / 08$ & 23.69 & 121 & $30 \pm 8$ & 0.20 & $337 \pm 3$ & $56 \pm 4$ & $28 \pm 3$ & 0.488 & 7.28 & B1 \\
\hline 252 & $00 / 0$ & 24.08 & & $15 \pm 4$ & .24 & $18 \pm 7$ & $57 \pm 4$ & $-10 \pm 3$ & 0.418 & 3.05 & B1 \\
\hline 253 & $00 / 09$ & 24.09 & & $26 \pm 5$ & & $213 \pm 7$ & $45 \pm 3$ & $71 \pm 9$ & 465 & 8.71 & B1 \\
\hline 254 & $00 / 0$ & 24.15 & & $27 \pm 6$ & & $103 \pm 8$ & $59 \pm 3$ & $157 \pm 3$ & 0.499 & 4.24 & B1 \\
\hline 255 & $00 / 0$ & 24.39 & & $22 \pm 5$ & .20 & $76 \pm 3$ & $41 \pm 3$ & $14 \pm 12$ & 0.436 & 18.29 & B2 \\
\hline 256 & $00 / 0$ & 24.36 & & $20 \pm 8$ & .21 & $4 \pm 7$ & $69 \pm 3$ & & 474 & 5.52 & B1 \\
\hline 257 & $00 / 09 / 16$ & 23.07 & & $12 \pm 5$ & .20 & $313 \pm 3$ & $30 \pm 3$ & $-95 \pm 5$ & 0.549 & 0.80 & $\mathrm{Cl}$ \\
\hline 258 & 00/09/16/23:C & 23.92 & 122.50 & $26 \pm 4$ & 0.25 & $320 \pm 6$ & $23 \pm 10$ & $162 \pm 3$ & 0.516 & 4.10 & $\mathrm{Cl}$ \\
\hline 59 & 00/09/20/13:2 & 24.73 & 121.71 & $86 \pm 11$ & $4.04 \pm 0.20$ & $244 \pm 9$ & $38 \pm 5$ & $92 \pm 3$ & 0.322 & 2.77 & B2 \\
\hline 260 & $00 / 09 / 21 / 06$ & 22.07 & 121.48 & $45 \pm 11$ & $4.56 \pm 0.23$ & $337 \pm 7$ & $44 \pm 6$ & $80 \pm 12$ & 0.562 & 9.33 & $\mathrm{Cl}$ \\
\hline 261 & $00 / 09 / 26 / 17: 16: 39.4$ & 23.36 & 120.02 & $13 \pm 5$ & $4.16 \pm 0.21$ & $324 \pm 5$ & $49 \pm 3$ & $-10 \pm 10$ & 0.586 & 10.44 & $\mathrm{C} 2$ \\
\hline 262 & 00/09/29/23:41:23.0 & 23.06 & 120.85 & $34 \pm 6$ & $4.20 \pm 0.21$ & $252 \pm 3$ & $36 \pm 4$ & $130 \pm 4$ & 0.571 & 1.29 & $\mathrm{Cl}$ \\
\hline 263 & 00/10/04/20:29:22.5 & 24.12 & 121.56 & $27 \pm 7$ & $4.16 \pm 0.21$ & $50 \pm 11$ & $42 \pm 3$ & $104 \pm 13$ & 0.510 & 0.90 & $\mathrm{Cl}$ \\
\hline 264 & 00/10/06/19:09:15.1 & 23.63 & 120.85 & $15 \pm 5$ & $3.97 \pm 0.20$ & $336 \pm 3$ & $40 \pm 7$ & $14 \pm 8$ & 0.480 & 0.00 & B1 \\
\hline 265 & 00/10/24/07:11:57.7 & 23.90 & 121.53 & $28 \pm 6$ & $4.47 \pm 0.22$ & $217 \pm 3$ & $44 \pm 8$ & $91 \pm 3$ & 0.438 & 7.60 & B1 \\
\hline 266 & 00/10/30/19:54:05.6 & 24.24 & 121.05 & $14 \pm 6$ & $3.86 \pm 0.19$ & $269 \pm 6$ & $43 \pm 7$ & $-122 \pm 6$ & 0.514 & 12.24 & $\mathrm{C} 2$ \\
\hline 267 & 00/11/20/00:07:09.4 & 24.79 & 121.91 & $17 \pm 6$ & $4.35 \pm 0.22$ & $30 \pm 6$ & $36 \pm 8$ & $-112 \pm 6$ & 0.532 & 24.39 & $\mathrm{C} 2$ \\
\hline 268 & $00 / 11 / 23 / 11: 22: 12.4$ & 23.94 & 122.49 & $30 \pm 6$ & $4.03 \pm 0.20$ & $318 \pm 9$ & $37 \pm 5$ & $154 \pm 5$ & 0.498 & 15.57 & B2 \\
\hline 269 & 00/11/29/11:00:33.0 & 23.86 & 121.77 & $24 \pm 8$ & $4.83 \pm 0.24$ & $254 \pm 6$ & $36 \pm 4$ & $149 \pm 12$ & 0.459 & 5.27 & B1 \\
\hline 270 & 00/11/29/13:41:40.7 & 23.86 & 121.77 & $26 \pm 7$ & $4.15 \pm 0.21$ & $256 \pm 9$ & $55 \pm 5$ & $162 \pm 7$ & 0.451 & 4.40 & B1 \\
\hline 271 & 00/12/10/10:08:38.6 & 23.11 & 120.21 & $18 \pm 4$ & $4.36 \pm 0.22$ & $132 \pm 4$ & $70 \pm 10$ & $9 \pm 3$ & 0.566 & 0.28 & $\mathrm{C} 1$ \\
\hline 272 & $00 / 12 / 10 / 19: 30: 44.5$ & 23.12 & 120.23 & $26 \pm 5$ & $4.95 \pm 0.25$ & $134 \pm 3$ & $78 \pm 11$ & $12 \pm 9$ & 0.535 & 6.23 & $\mathrm{C} 1$ \\
\hline 273 & $00 / 12 / 12 / 20: 32: 52.7$ & 23.97 & 122.68 & $27 \pm 7$ & $5.19 \pm 0.26$ & $262 \pm 3$ & $21 \pm 3$ & $98 \pm 4$ & 0.521 & 0.28 & $\mathrm{C} 1$ \\
\hline 274 & $00 / 12 / 22 / 11: 23: 00.4$ & 24.08 & 121.15 & $12 \pm 5$ & $4.20 \pm 0.21$ & $118 \pm 4$ & $66 \pm 8$ & $-162 \pm 6$ & 0.512 & 4.08 & $\mathrm{C} 1$ \\
\hline 275 & 00/12/28/09:01:26.3 & 23.44 & 122.04 & $31 \pm 6$ & $4.72 \pm 0.24$ & $334 \pm 3$ & $56 \pm 3$ & $19 \pm 3$ & 0.409 & 0.24 & B1 \\
\hline 276 & 00/12/29/18:03:28.6 & 24.36 & 121.88 & $22 \pm 4$ & $4.76 \pm 0.24$ & $105 \pm 10$ & $49 \pm 5$ & $19 \pm 10$ & 0.540 & 7.10 & $\mathrm{C} 1$ \\
\hline 211 & & 24.19 & 122.30 & $45 \pm 6$ & & & $40 \pm 3$ & $130 \pm 6$ & 0.443 & 0.21 & D1 \\
\hline
\end{tabular}

${ }^{1}$ A complete table with individual moment tensor components can be found in BATS website at http://bats.earth.sinica.edu.tw/CMT_Solutions

${ }^{2}$ Origin time (Year/Month/Day/hr:min:sec) and epicentral locations $\left({ }^{\circ} \mathrm{N},{ }^{\circ} \mathrm{E}\right)$ are those reported by the Seismology Center, Central Weather Bureau, Taiwan.

${ }^{3}$ Best double-couple solutions (units in degrees).

${ }^{4}$ See Table 2 for definitions of $E$ and $\varepsilon$. 
To characterize the quality of inversion, we organize each result by assigning a letter (A$F)$ and a number (1-4) based on the value of two parameters: the waveform misfit (E) and the amount of CLVD component $(\varepsilon)$, respectively. Thus, $\mathrm{A} 1$ and $\mathrm{F} 7$ respectively represent the best and worst possible quality. Table 2 shows the range of $E$ and $\varepsilon$ that defines each category (Kao et al. 1998; Kao and Jian, 1999).

\section{RESULTS}

Following previous work by Kao and Jian (1999), we only report source parameters if (1) 3-component waveforms from at least three stations are available for inversion, and (2) the quality of result are higher than C4. Table 1 shows the results of 277 earthquakes that satisfy these conditions and Figs 2-8 show the corresponding best double-couple solutions with focal depths. Only 36 events occurred before the Chi-Chi mainshock, and most of the remaining 241 events are aftershocks of the Chi-Chi sequence.

For brevity, full results of inversion including observed and synthetic waveforms appear as an appendix (in electronic form) of this article and are available at the BATS web site (http:/ /bats.earth.sinica.edu.tw/CMT_Solutions/cmtF1999.html and http://bats.earth.sinica.edu.tw/ CMT_Solutions/cmtF2000.html). Corresponding tables (including Table 1 of this study and those for earthquakes between 1995 and 1998) are also available at the same site (http://bats. earth.sinica.edu.tw/CMT_Solutions).

In this section, we briefly discuss the results in seven periods (Figs 2-8) with each corresponding to a natural break in the pattern of seismicity. For the Chi-Chi sequence and the concentrated seismicity beneath the southwestern fold-and-thrust belt, Kao and Chen (2000) and Chen and Kao (2000a and b) discussed the overall spatial patterns of earthquakes in detail, which include newly discovered seismogenic structures, kinematics of faulting, and tectonic significances.

Table 2. Classification Describing the Quality of Results.

\begin{tabular}{cc} 
Class & Criteria \\
\hline & Average Waveform Misfit (E) \\
A & $0 \leq \mathrm{E}<0.3$ \\
B & $0.3<\mathrm{E} \leq 0.5$ \\
C & $0.5<\mathrm{E} \leq 0.7$ \\
D & $0.7<\mathrm{E} \leq 0.9$ \\
E & $0.9<\mathrm{E} \leq 1.1$ \\
F & $\mathrm{E}>1.1$ \\
& Fraction of CLVD component ( $)$ \\
1 & $\varepsilon \leq 0.1$ \\
2 & $0.1<\varepsilon \leq 0.25$ \\
3 & $0.25<\varepsilon \leq 0.4$ \\
4 & $\varepsilon>0.4$ \\
\hline
\end{tabular}




\subsection{Time Period Before the Main Shock of the Chi-Chi Sequence (1/1/1999 to 9/20/1999)}

Our results reveal two regions of frequent seismicity: one is off the eastern seaboard, particularly in a region between the southernmost Ryukyu arc and NE Taiwan. The other is beneath southwestern Taiwan adjacent to the southern end of the surface rupture during the Chi-Chi sequence. In contrast, the entire central and northern Taiwan was seismically quiescent with only one significant event beneath the Central Range (No. 14).

Focal depths of all onshore events are shallower than $25 \mathrm{~km}$ (shown by crosses in Fig. 2), whereas a few offshore earthquakes occurred at much greater depths, and are likely to be within the subducted Philippine Sea slab (e.g., No. 7 at 102 km, Fig. 2). The majority of events are thrust faulting. Earthquakes associated with normal faulting are either related to the opening of the Okinawa trough (e.g., No. 13), or within the Manila trench-Luzon arc subduction system (e.g., No. 12 and 22).

\subsection{The First Three Days of the Chi-Chi Sequence (9/20/1999 to 9/22/1999)}

The source parameters of Chi-Chi mainshock have been studied in detail using teleseismic body waveform inversion (Kao and Chen, 2000), and are not included in the present report. At the onset of this sequence, nearly all aftershocks occurred to the east of the surface rupture (the Chelungpu fault). However, the region immediately to the east of the fault, where several meters of slip occurred during the main shock, lacked any significant aftershocks (Fig. 3). The events occurred north of $\sim 23.7^{\circ} \mathrm{N}$ seem to form two $\mathrm{N}-\mathrm{S}$-trending bands that delineate a curious aseismic zone beneath the Central Range. Background seismicity prior to 1999 had already defined this aseismic zone (e.g., Wang et al, 1994), a persistent feature even the Chi-Chi sequence did not breach (Chen and Kao, 2000a).

A cluster of aftershocks south of $\sim 23.7^{\circ} \mathrm{N}$ extends NNW-SSE from the southern terminus of the Chelungpu fault into the Central Range (Fig. 3). Most of these events show strike-slip faulting. Kao and Chen (2000) and Chen and Kao (2000a) interpreted this pattern reflecting slip on tear fault(s) that marks the southern end of the west-verging Chelungpu thrust system.

To the east of the aseismic zone and beneath the eastern flank of the Central Range, several events of normal faulting mechanism occurred at depths shallower than $10-15 \mathrm{~km}$ (e.g., No. 41, 53, and 73). Events at greater depths ( 20 km and more), on the other hand, consistently showed thrust faulting (e.g., No. 69 and 76; Fig. 3).

\subsection{The Next Week (9/23/1999-9/30/1999)}

In this time period (Fig. 4), the basic pattern of seismicity is similar to that shown in Fig. 3 except that (1) the footwall of the Chelungpu fault began to show some seismicity (No. 93, 101, and 110), and (2) both the northem and southern ends of the Chelungpu fault zone showed concentrated aftershocks. The region immediately to the east of the surface rupture remains absent of aftershocks except at the southernmost tip of the rupture where the strike bends from N-S to NE-SW.

Notice that aftershocks reflecting strike-slip faulting are now abundant along both northern and southern boundaries of the Chelungpu thrust block (Fig. 4), further substantiate the 


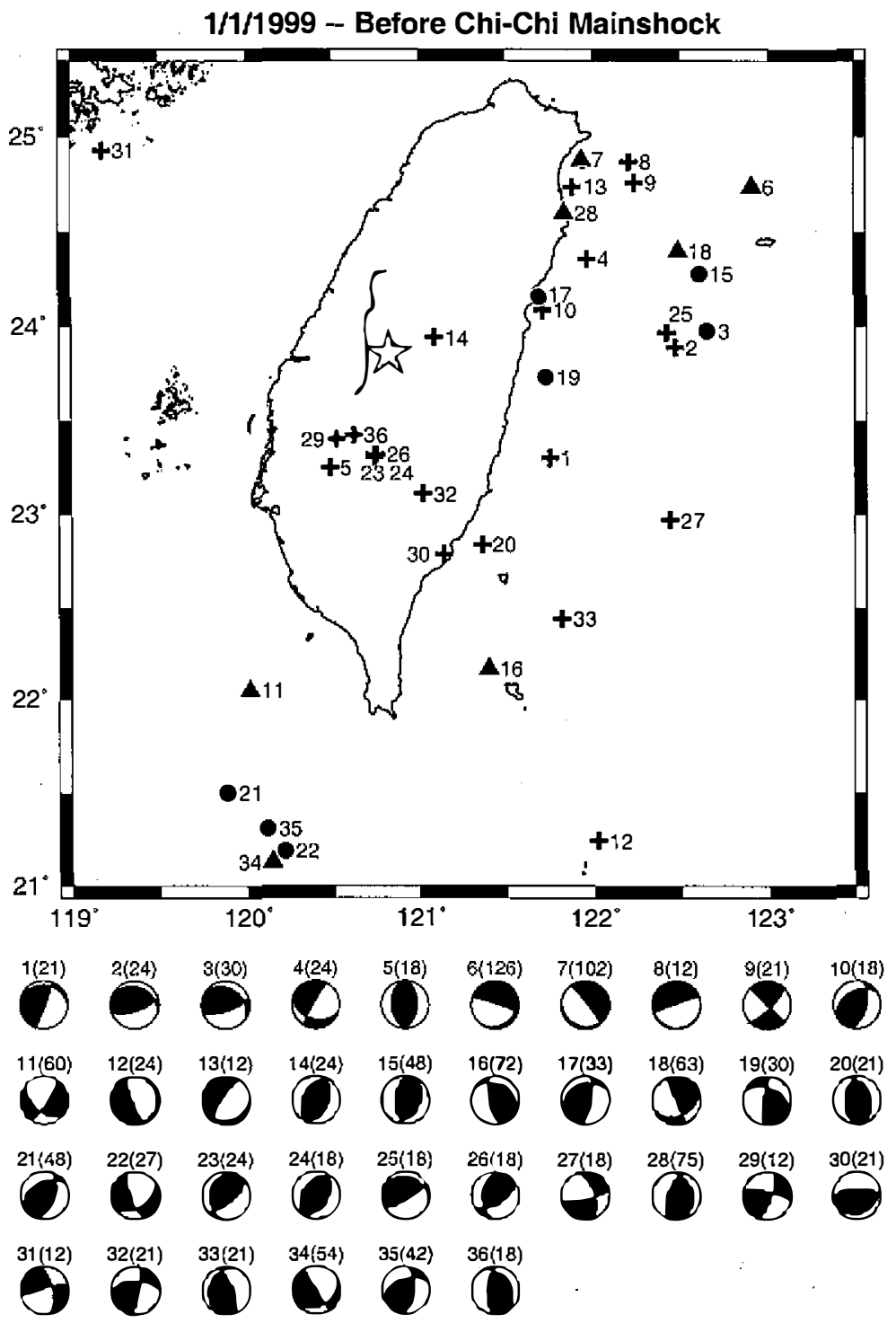

Fig. 2. Epicenters and source parameters of regional earthquakes that occurred in 1999 before the Chi-Chi sequence. Event numbers correspond to those in Table 1. Symbols show different ranges of focal depths: crosses for 0$25 \mathrm{~km}$, solid dots for $25-50 \mathrm{~km}$, and solid triangles for $>50 \mathrm{~km}$. For reference, the open star and solid curve mark the epicenter of the main shock and the surface break (i.e., the Chelungpu fault), respectively. The corresponding focal mechanisms (lower-hemisphere projection with darkened areas showing quadrants with compressional $P$ wave first motions) appear below the map. Above each fault plane solution, we show the event number and the focal depth (in parenthesis, unit in $\mathrm{km}$ ). 


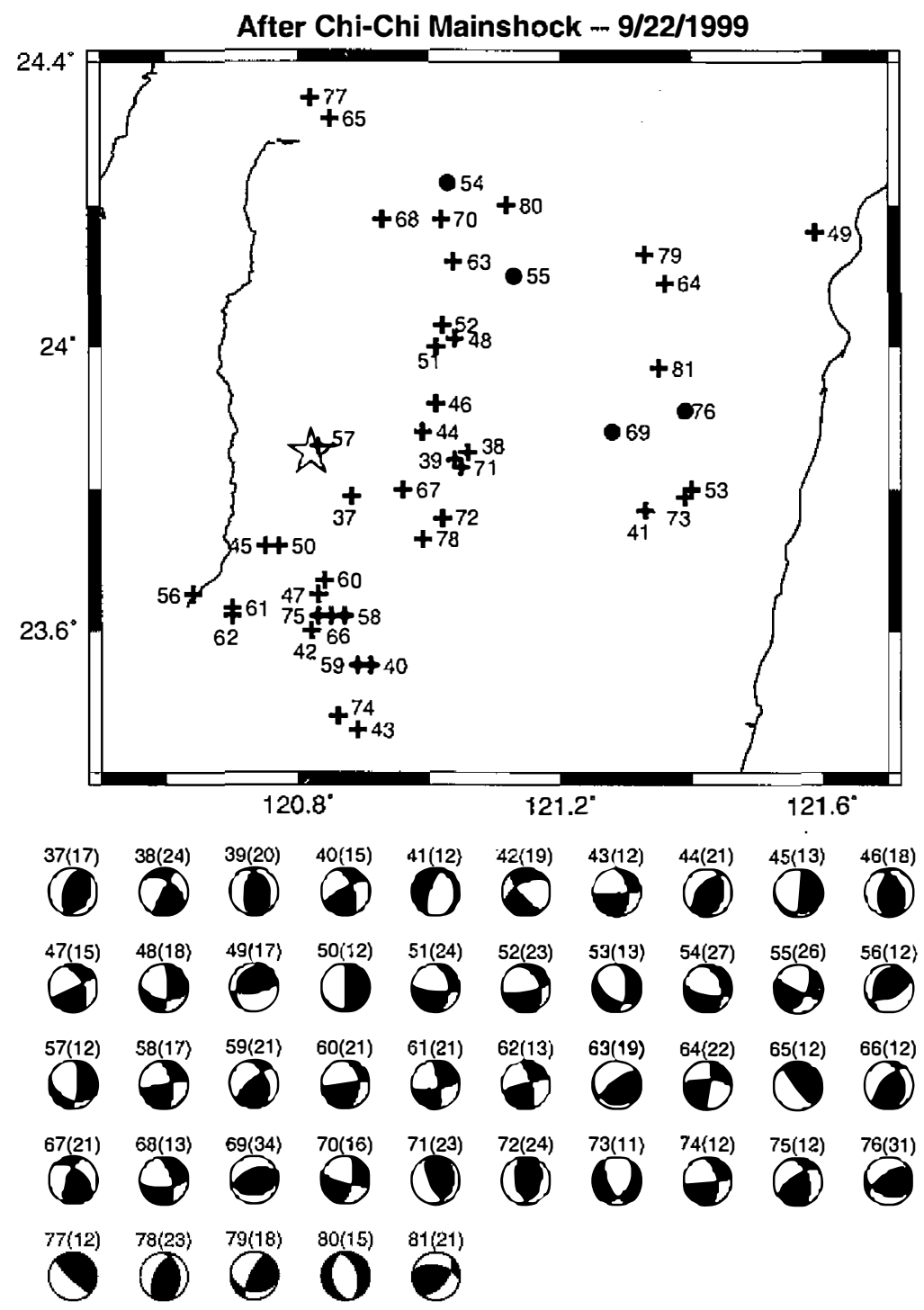

Fig. 3. Epicenters and source parameters of regional earthquakes that occurred within the first two days after the Chi-Chi mainshock (up to 9/22/1999). Layout is the same as that in Fig. 2.

role of tear faults as termination of this thrust system.

Under the eastern flank of the Central Range, two interesting pairs of events (No. 87 and 88 , No. 106 and 107) confirmed the change from normal faulting on top (focal depths $\sim 12-15$ $\mathrm{km}$ ) to thrust faulting at depth (focal depths $\sim 27-28 \mathrm{~km}$ ). Although the physics for such a systematic variation is not immediately clear, there is little doubt that the state of strain in upper crust is distinct from that at deeper depth beneath the eastern Central Range. 


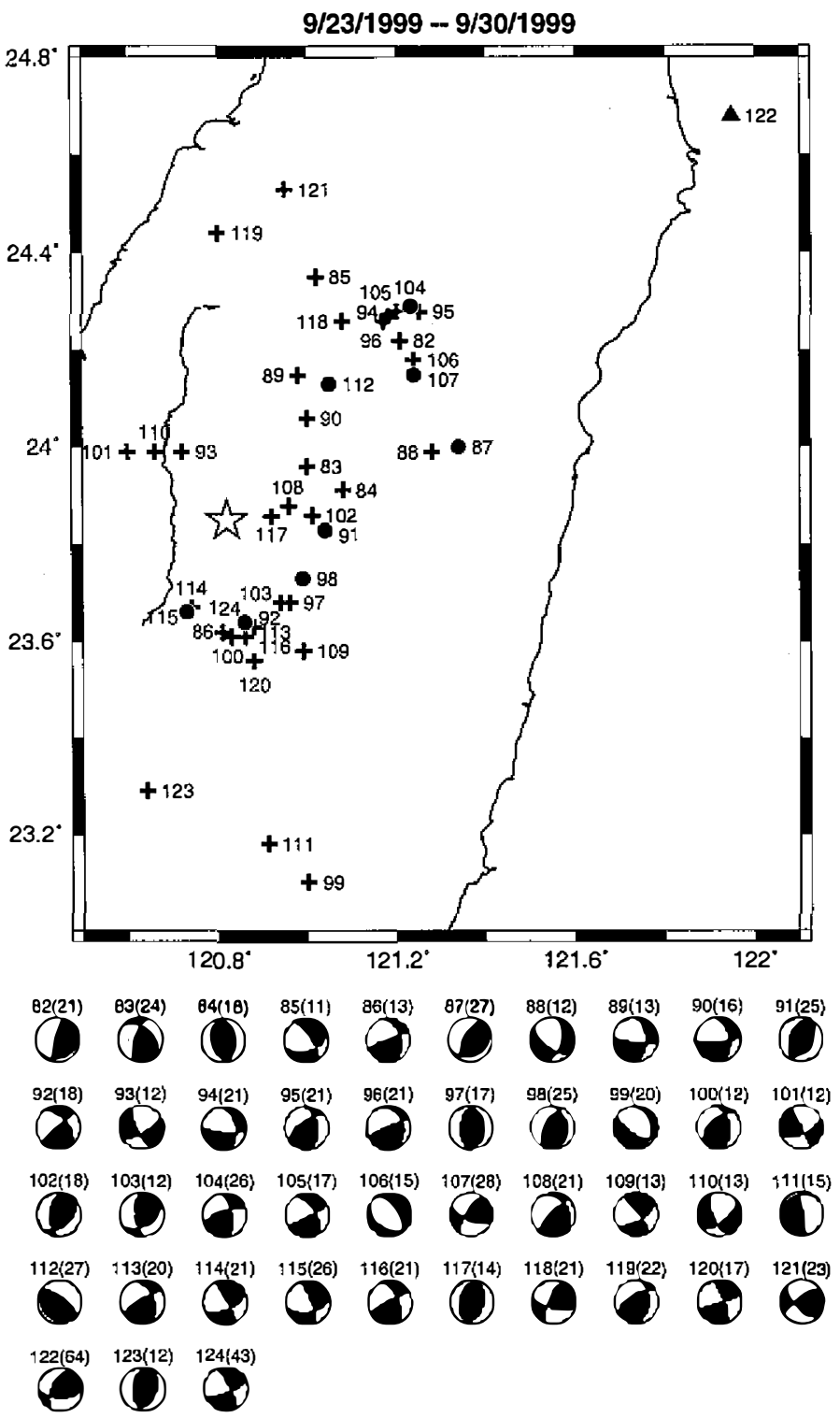

Fig. 4. Epicenters and source parameters of regional earthquakes that occurred during 9/23/1999-9/30/1999. Layout is the same as that in Fig. 2.

\subsection{New Activity in Southwestern Taiwan (10/1/1999-11/17/1999)}

The entire northern Taiwan including the northern half of the Chelungpu thrust system was seismically quiet during this time period. Most aftershocks of the Chi-Chi sequence scattered south of $\sim 24^{\circ} \mathrm{N}$ (Fig. 5). Starting on October 22, 1999, a burst of events occurred southwest of the source zone of the Chi-Chi sequence (starting with No. 150, ending with No. 173; 


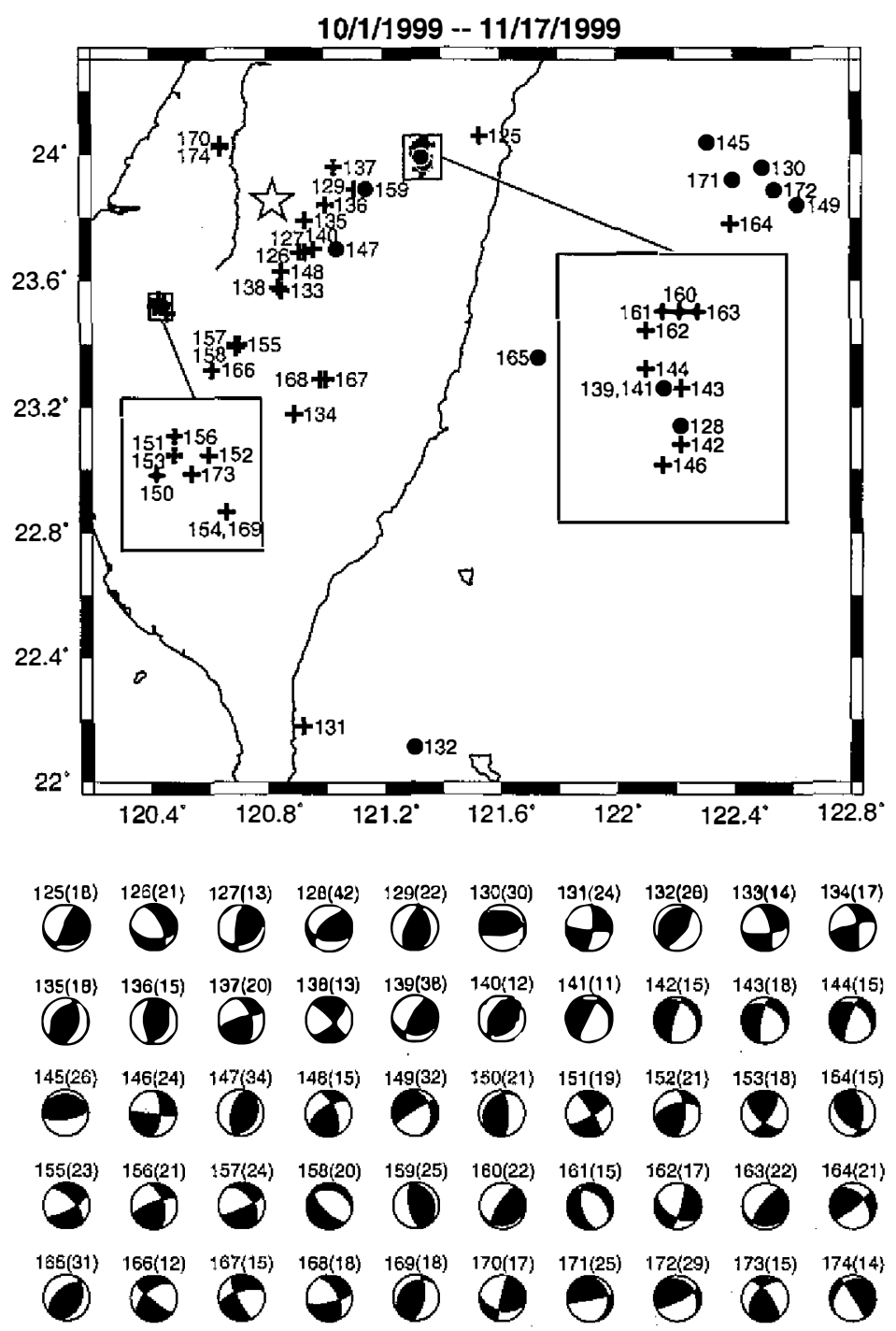

Fig. 5. Epicenters and source parameters of regional earthquakes that occurred during 10/1/1999-1 1/17/1999. Layout is the same as that in Fig. 2.

Fig. 5). Earthquakes in this cluster showed either thrust or strike-slip mechanisms with most $P$-axes associated with high-angle reverse faulting beneath the southwestern foothills, a tectonic domain distinct from the source region of the Chi-Chi sequence.

Another cluster of earthquakes occurred beneath the east flank of the Central Range (Fig. 5). Similar to the previous time period, all earthquakes occurred at depths less than $\sim 20 \mathrm{~km}$ (No. 141-144, 161, and 162) showed normal faulting, whereas most deeper events reflected thrust (No. 128, 139, 160, and 163) or strike-slip (No. 146) faulting. 
A number of events occurred $\sim 80 \mathrm{~km}$ off the east coast (No. 130, 145, 149, 164, 171, and 173; Fig. 5). With only one exception, these events show low-angle thrust faulting. The shallow-dipping nodal plane trends E-W to NE-SW, which are sub-parallel to the local strike of the southernmost Ryukyu subduction zone. Thus these earthquakes must have occurred along the interplate thrust zone between the subducting Philippine Sea slab and the Eurasian plate.

\subsection{Resurgence of the Ch-Chi Sequence (11/18/1999-6/30/2000)}

In contrast to the previous time period, seismicity intensified once again in the central and northem portions of the aftershock zone of the Chi-Chi sequence (Fig. 6). The central cluster (No. 188, 203, 218, 219, 221-223) includes a large (Mw 6.1) event that occurred at the depth

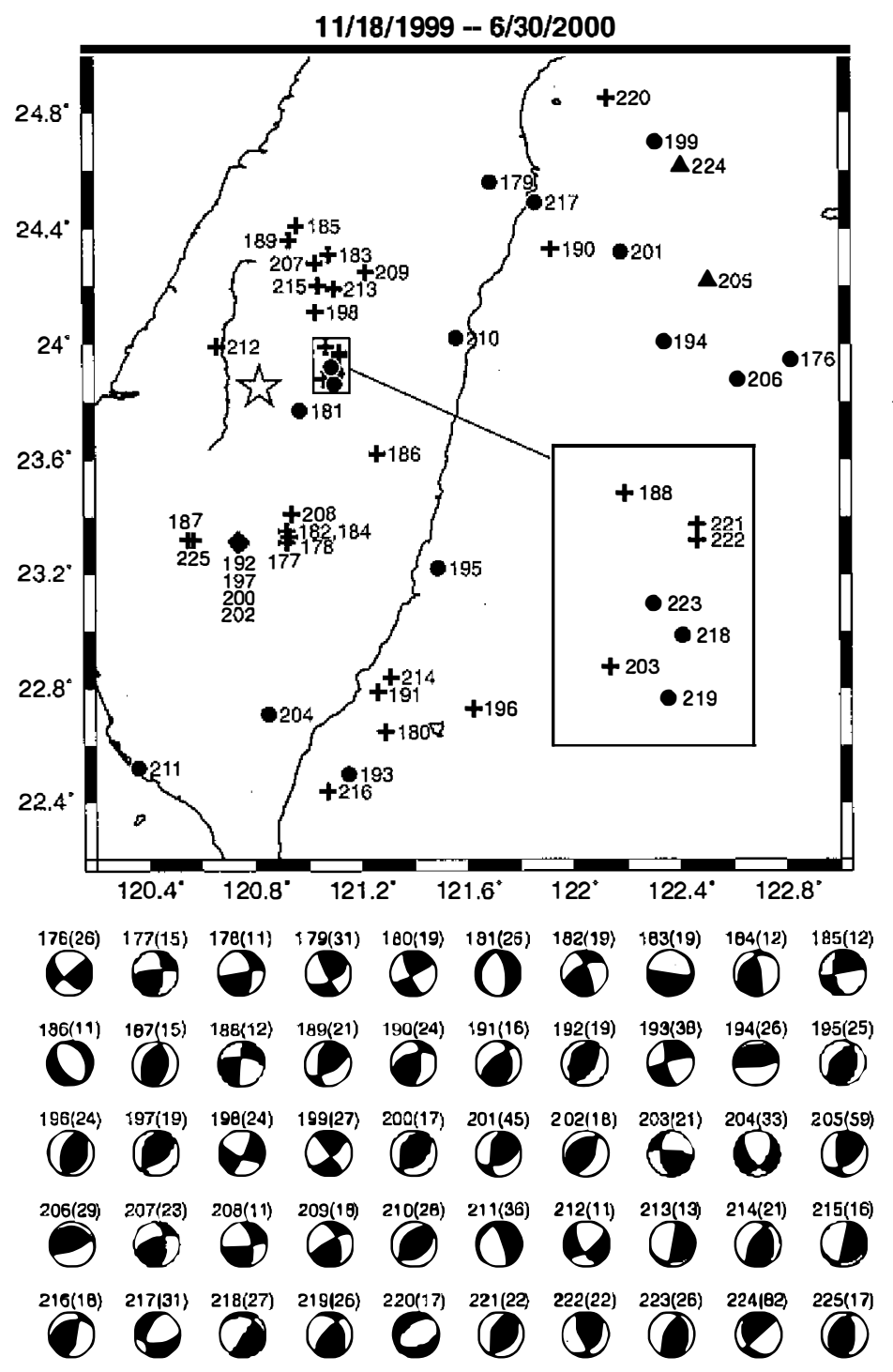

Fig. 6. Epicenters and source parameters of regional earthquakes that occurred during $11 / 18 /$ 1999-6/30/2000. Layout is the same as that in Fig. 2. 
of $27 \mathrm{Km}$ on June 10, 2000 (No. 218). In fact, both layers of the dual, out-of-sequence thrust remained active at this late stage of the Chi-Chi sequence leaving little doubt about the importance of the deeper layer in seismogenesis and tectonics (Kao and Chen, 2000).

Meanwhile, high activity continued beneath the southwestern fold-and-thrust belt (No. 187 and 225; No. 192, 197, 200, and 202; No. 178, 182, 184, and 208; Fig. 6) with the same pattern of faulting as in previous time periods.

Background seismicity is also high off the eastern shore (Fig. 6). All earthquakes seem to reflect known patterns of active deformation such as: normal faulting (No. 220) in the opening of the Okinawa trough, downdip extensional of the subducting Philippine Sea plate at intermediate-depths (No. 205 and 224), and interplate slip and shortening of the fore-arc region along the Ryukyu and Luzon arcs (e.g., No. 190, 194, 201, 206; No. 180, 191, 193, 214, 216, respectively).

\subsection{The Waning Chi-Chi Sequence (7/1/2000-8/31/2000)}

The pattern of regional seismicity in this time period is no longer dominated by aftershocks of the Chi-Chi sequence (Fig. 7). Instead, most noticeable activity occurred offshore, immediately to the east of Hualien $\left(\sim 23.6-24^{\circ} \mathrm{N} ;\right.$ Fig. 7$)$. Nearly all these events show thrust faulting, which are consistent with vigorous $\mathrm{E}-\mathrm{W}$ collision in this region.

As in previous periods, the shallow portion of the eastern Central Range is characterized by normal faulting with T-axes sub-parallel to the overall structural trend of NE-SW (No. 230, 246-249). Another normal faulting event marks the opening near the westem terminus of the Okinawa trough (No. 228).

\subsection{Epilogue (9/1/2000-12/31/2000)}

In the last three months of 2000 , the Hualien area and the region farther to the east remained to be the most active region (Fig. 8). Earthquake faulting continued to reflect known active tectonics (e.g., Kao et al. 1998; Kao and Jian 2001): normal faulting in the Okinawa trough (No. 267), downdip extension within subducted slab (No. 259), low-angle thrust faulting along the plate interface (No. 258, 268, and 273), and lateral compression between the subducted Philippine Sea slab and Eurasia (i.e., slab-continental collision, Kao and Jian 2001; No. 253, 254, 263, 265, 269, 270, and 277).

One lone event occurred off the southwestern coastline (No. 261). Its mechanism of oblique strike-slip faulting indicated N-S compression or equivalently E-W extension. Since EW/NW-SE compression dominates the western Taiwan orogen, and an $\mathrm{m}_{\mathrm{v}} \sim 6.5$ normal faulting earthquake consistent with N-S extension occurred inside the Taiwan Strait in 1994 (e.g., Kao and Wu 1996), event No. 261 may have occurred in a ransition zone between contraction to extension regimes of strain (i.e., strain permutation, $\mathrm{Hu}$ et al. 1997).

\section{SPECIAL DATA RELEASE FROM BATS: BROADBAND WAVEFORMS FROM THE CHI-CHI EARTHQUAKE SEQUENCE}

DMC-IES hereby announces that BATS waveform data for the entire Chi-Chi earthquake 


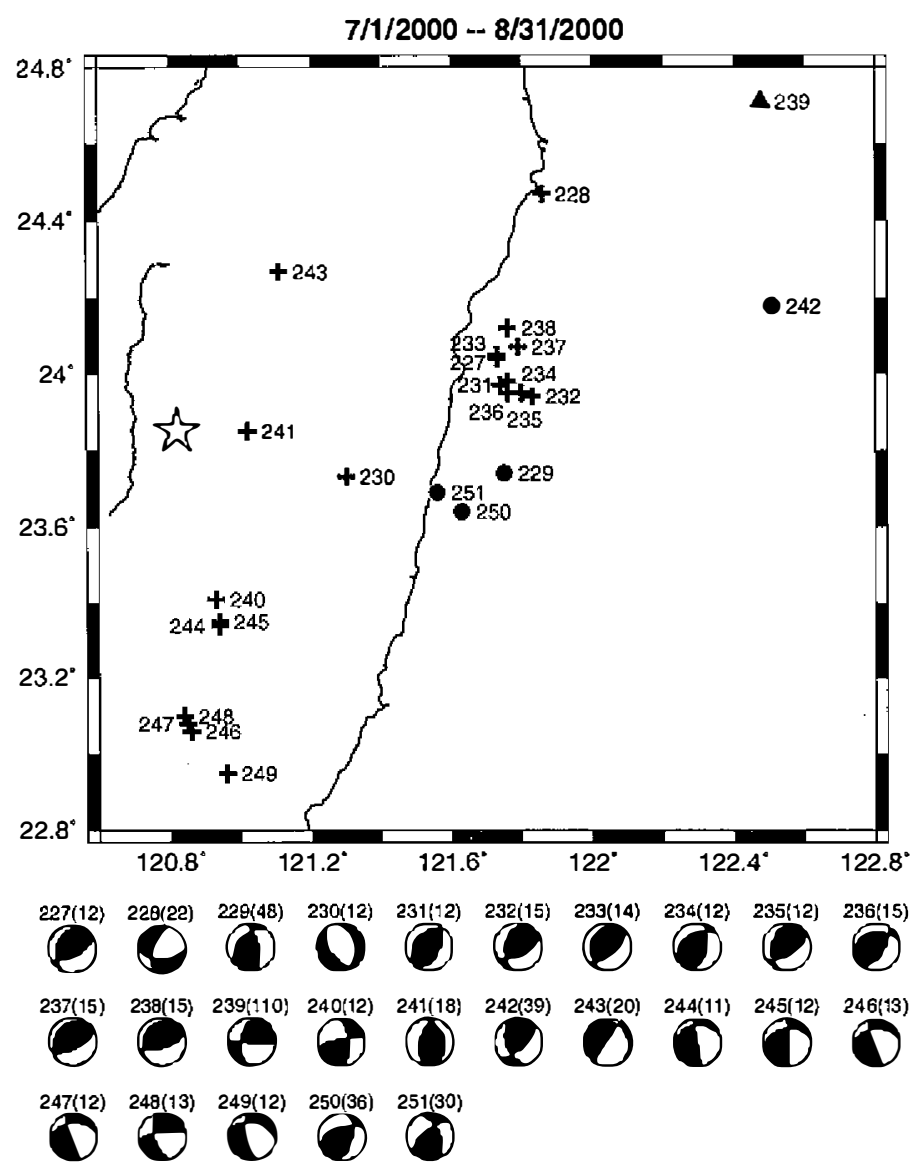

Fig. 7. Epicenters and source parameters of regional earthquakes that occurred during 7/1/2000-8/31/2000. Layout is the same as that in Fig. 2.

sequence are now freely available. For the user's convenience, we have prepared a CD-ROM of event-oriented files with our results of inversion (both graphics and tables) and original waveforms recorded by BATS for 170 aftershocks of the sequence shown in Table 1 . These 170 events occurred within one year after the main shock and fell between events No. 36 and 257.

For each event in the CD-ROM, we performed quality control on available BATS stations and then extract a 15-minutes segment of data from each broadband channel, commence at $300 \mathrm{~s}$ before and terminate at $600 \mathrm{~s}$ after the first $\mathrm{P}$ arrival. Together with the corresponding instrument and network information, we then create a SEED volume by combining all extracted waveforms for each event. Depending on the number of available waveforms, the file size for each event varies from $\sim 0.9$ to $\sim 2.5 \mathrm{MB}$.

This dataset on CD-ROM and an electronic version of Tables 1-2 and Figs 2-8 are also readily available through the Internet at the BATS website (http://bats.earth.sinica.edu.tw/ Chi-Chi_CMT). 


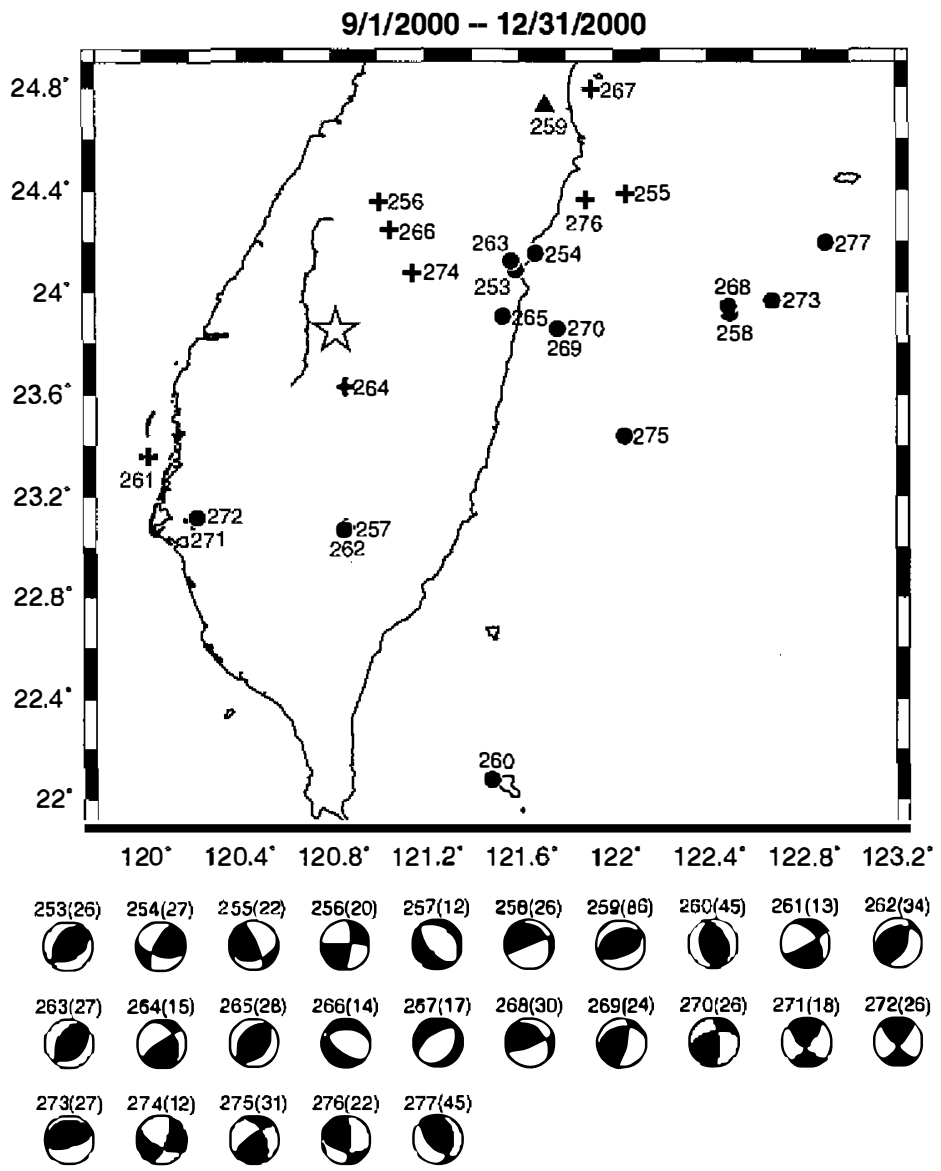

Fig. 8. Epicenters and source parameters of regional earthquakes that occurred during 9/1/2000-12/31/2000. Layout is the same as that in Fig. 2.

\section{SUMMARY}

We report a systematic study of centroid-moment-tensor (CMT) solutions including refined focal depths from our previous reports for regional earthquakes that occurred near Taiwan during 1999-2000. We primarily used waveform data recorded by the Broadband Array in Taiwan for Seismology (BATS) in the inversion. For the largest events, our results have been corroborated by the inversion of teleseismic broadband data (Kao and Chen 2000; Chen and Kao 2000a, 2000b). Among a total of 277 earthquakes in this report, most of them belong to the Chi-Chi earthquake sequence except the 36 events occurred prior to the main shock of this sequence.

To improve estimations on uncertainty of focal depths, we performed inversion at a refined interval of $1 \mathrm{~km}$ for focal depth for all events that occurred after the Chi-Chi mainshock. As in previous reports (e.g., Kao et al. 1998), we make realistic estimations of uncertainties by searching the model space through forward modeling. 
Nine months prior to the Chi-Chi earthquake, the entire central and northern Taiwan was seismically quiet with only one significant event beneath the Central Range. Immediately after the mainshock, significant aftershocks of the Chi-Chi sequence occur only along the east side of the source zone of the mainshock that broke the surface along the Chelungpu thrust. Aftershocks associated with strike-slip faulting concentrate near the northern and southern ends of the surface rupture, marking tear faults that accommodate the main west-verging thrust block. The aftershocks then spread to the footwall as the tear faults continue to slip. Intriguingly, the high-level aftershocks associated with the Chi-Chi sequence never breached an aseismic region under the Central Range known from background seismicity.

In October and November of 1999, the northern segment of the Chelungpu fault system and the entire northern Taiwan became seismically quiet. Meanwhile, a cluster of events (with both thrust and strike-slip faulting) took place beneath the southwestern fold-and-thrust belt farther to the south and west of the rupture zone of the Chi-Chi sequence.

In this period, a curious pattern of earthquakes continued to appear beneath the east flank of the Central Range where earthquakes reflected normal faulting at depths less than about 20 $\mathrm{km}$ but showed reverse faulting at greater depths down to almost $40 \mathrm{~km}$.

Starting in December of 1999, aftershocks of the Chi-Chi sequence diminished while background seismicity increased, reflecting a wide variety of faulting that are known to occur in each of the complex tectonic domains in and around Taiwan.

To facilitate further research, DMC-IES has compiled a CD-ROM containing all the results discussed here, plus original waveforms of 170 aftershocks of the Chi-Chi sequence recorded by BATS within one year from the main shock. The waveform files are prepared in SEED format of the FDSN and the CD-ROM is available to the entire community upon request. All information in this article and the entire content of the CD-ROM are also publicly accessible at the BATS website (http://bats.earth.sinica.edu.tw/Chi-Chi_CMT). At the same website, readers who are interested in BATS data not included in this CD-ROM can contact the DMCIES for further assistance.

Acknowledgments We thank technical staff at the IES, led by Chun-Chi Liu, who assumes the primary responsibility for maintaining BATS. The manuscript benefits from comments by How-Wei Chen and an anonymous reviewer. This research was partially supported by the National Science Council, Republic of China, under grants NSC89-2921-M-001-012-EAF and NSC90-2119-M-001-011. Work by Chen is partially support by the US National Science Foundation (NSF) under Grant No. EAR-01-06786.

\section{REFERENCES}

Chen, W.-P., and H. Kao, 2000a: Evidence for dual, out-of-sequence thrust faulting during the Chi-Chi (Taiwan) earthquake sequence of 1999. Int. Workshop on Annual Commemoration of the Chi-Chi Earthquake, 71-81.

Chen, W.-P., and H. Kao, 2000b: Seismogenic reverse faulting in southwestem Taiwan: Inversion tectonics at mid-crustal depths (Abstract). EOS Trans. Am. Geophys. Union, 81, F883. 
Dreger, D. S., and D. V. Helmberger, 1993: Determination of source parameters at regional distances with three-component sparse network data. J. Geophys. Res., 98, 8107-8125.

Dziewonski, A. M., T.-A. Chou, and J. H. Woodhouse, 1981: Determination of earthquake source parameters from waveform data for studies of global and regional seismicity. $J$. Geophys. Res., 86, 2825-2852.

Hu, J.-C., J. Angelier, and S.-B. Yu, 1997: An interpretation of the active deformation of southem Taiwan based on numerical simulation and GPS studies. Tectonophysics, 274, 145-169.

Kao, H., and W.-P. Chen, 2000: The Chi-Chi earthquake sequence: Active, out-of-sequence thrust faulting in Taiwan. Science, 288, 2346-2349.

Kao, H., and F. T. Wu, 1996: The 16 September 1994 earthquake $(\mathrm{mb}=6.5)$ in the Taiwan strait and its tectonic implications. TAO, 7, 13-29.

Kao, H., and P.-R. Jian, 1999: Source parameters of regional earthquakes in Taiwan: July 1995-December 1996. TAO, 10, 585-604.

Kao, H., and P.-R. Jian, 2001: Seismogenic patterns in the Taiwan region: Insights from source parameter inversion of BATS data. Tectonophysics, 333, 179-198.

Kao, H., P.-R. Jian, K.-F. Ma, B.-S. Huang, and C.-C. Liu, 1998: Moment-tensor inversion for offshore earthquakes east of Taiwan and their implications to regional collision. Geophys. Res. Lett., 25, 3619-3622.

Kao, H., Y.-H. Liu, and P.-R. Jian, 2001: Source parameters of regional earthquakes in Taiwan: January-December 1997. TAO, 12, 431-439.

Kawakatsu, H., 1995: Automated near-realtime CMT inversion. Geophys. Res. Lett., 22, 25692572.

Lay, T., J. Ritsema, C. J. Ammon, and T. Wallace, 1994: Rapid source-mechanism analysis of the April 29, 1993 Cataract Creek (Mw = 5.3), northern Arizona earthquake. Bull. Seism. Soc. Am., 84, 451-457.

Pasyanos, M. E., D. S. Dreger, and B. Romanowicz, 1996: Toward real-time estimation of regional moment tensors. Bull. Seism. Soc. Am., 86, 1255-1269.

Sipkin, S. A., 1982: Estimation of earthquake source parameters by the inversion of waveform data: synthetic waveforms. Phys. Earth Planet. Inter., 30, 242-259.

Takeuchi, N., S. Watada, S. Tsuboi, Y. Fukao, M. Koybayashi, Y. Matsuzaki, and T. Nakashima, 2002: Application of distributed object technology to seismic waveform distribution. Seism. Res. Lett., in press.

Wang, J.-H., K.-C. Chen, and T.-Q. Lee, 1994: Depth distribution of shallow earthquakes in Taiwan. J. Geol. Soc. China, 37, 125-142.

Zhu, L., and D. V. Helmberger, 1996: Advancement in source estimation techniques using broadband regional seismograms. Bull. Seism. Soc. Am., 86, 1634-1641. 